\title{
Revisión narrativa de instrumentos de autoevaluación vocal en Chile
}

\author{
Narrative review of vocal self-assessment instruments in Chile
}

María Celina Malebrán B. ${ }^{1}$, Liliana Henríquez N. ${ }^{2}$, Francisco Contreras-Ruston ${ }^{3}$

\section{Resumen}

Los sujetos disfónicos no siempre presentan una adecuada percepción de su voz o de cómo las alteraciones vocales afectan a su entorno, modificando su calidad de vida. Los protocolos de auto evaluación vocal ayudan al clínico a percibir si el paciente disfónico se siente afectado por sus problemas vocales o no. Existen numerosos protocolos de auto evaluación vocal, sin embargo, pocos de estos están actualmente validados en Chile. Este trabajo corresponde a una revisión narrativa respecto de la validación de instrumentos de autoevaluación vocal en Chile. Se llevó a cabo una búsqueda bibliográfica en: Pubmed, Scielo y Bireme. Fueron empleados los siguientes descriptores: Chile, voz, autoevaluación, calidad de vida, estudios de validación, comparación transcultural y sus respectivos términos en inglés: Chile, voice, validation study, self-assessment, quality of life, crosscultural comparision. Dos restricciones fueron empleadas: las investigaciones debían estar publicadas en inglés y/o español, con fecha de publicación entre 2008 y 2020. Se encontraron 41 artículos, eliminándose 24 duplicados y 13 de contenidos no relacionados, resultando en 4 artículos analizados correspondientes a 3 instrumentos traducidos al español. El uso de protocolos de autoevaluación vocal complementa la evaluación clínica y objetiva de la voz, contribuyendo con la percepción del paciente, relevante para el tratamiento fonoaudiológico, sin embargo, la cantidad de protocolos de autoevaluación vocal en Chile con traducción, adaptación cultural y validación estandarizada continúa siendo escasa.

Palabras clave: Voz, Chile, estudios de validación, calidad de vida, autoevaluación, comparación transcultural.

\begin{abstract}
Dysphonic subjects do not always present an adequate perception of their voice, or of how vocal alterations affect their environment, modifying their quality of life. Vocal self-assessment protocols help the clinician to perceive whether the dysphonic patient feels affected by his vocal problems or not. There are numerous protocols for vocal self-assessment, however, few of these are currently validated in Chile. This work corresponds to a narrative review regarding the validation of vocal self-assessment instruments in Chile. The bibliographic search was conducted in the search sites: Pubmed, Scielo and Bireme. The following descriptors were used: Chile, voice, validation studies, cross-cultural comparison. Two restrictions were used: the research had to be published in English and/or Spanish, with a publication date between 2008 and 2020. A total of 41 articles were found, eliminating 24 duplicates and 13 of unrelated content, resulting in 4 articles analyzed corresponding to 3 instruments translated into Spanish. The use of vocal self-assessment protocols complements the clinical and objective assessment of the voice, contributing to the perception of the patient, relevant for speech therapy, however, the number of vocal self-assessment protocols in Chile with translation, cultural adaptation and standardized validation remains sparse.
\end{abstract}

Keywords: Voice, Chile, validation studies, quality of life, self-assessment, cross-cultural comparison.
${ }^{1}$ Escuela de Fonoaudiología, Facultad de Ciencias de la Salud, Universidad Católica Silva Henríquez. Santiago, Chile.

¿Escuela de Fonoaudiología Universidad Santo Tomás. Viña del Mar, Chile. ${ }^{3}$ Universidad de Valparaíso. San Felipe, Chile.

Los autores declaran no tener conflictos de interés.

Recibido el 20 de septiembre de 2020. Aprobado el 14 de marzo de 2021.

Correspondencia:

María Celina Malebrán B. Tocornal 303. Escuela de Fonoaudiología

Facultad de Ciencias de la Salud. Universidad Católica Silva Henríquez- Santiago, Chile.

Email: mmalebran@ucsh.cl; celinamalebranbmello@gmail. com 


\section{Introducción}

El concepto de salud es multidimensional y se relaciona con aspectos físicos, mentales y sociales. Los pacientes con problemas vocales frecuentemente comentan limitaciones de naturaleza física, emocional y profesional que afectan su calidad de vida. Pese a que este concepto no es nuevo en la historia de la medicina, en fonoaudiología, ha sido recientemente incorporado en el proceso terapéutico. La evaluación de la voz es un procedimiento multidimensional que se compone de evaluaciones objetivas y perceptivas, con o sin el uso de protocolos. En la práctica vocal estándar, los pacientes son valorados por un equipo multidisciplinario que consiste, al menos, de un otorrinolaringólogo (ORL) y un fonoaudiólogo ${ }^{1}$.

Tomando como contexto el marco de la Organización Mundial de la Salud (OMS), la American Speech and Hearing Association (ASHA) señala que la valoración vocal tiene como fin identificar y describir los efectos del desorden de la voz en las capacidades, desempeño y participación del individuo en los contextos comunicativos de la vida diaria ${ }^{2}$. Una de las formas de conseguir lo mencionado es mediante la aplicación de cuestionarios de autoevaluación para determinar el impacto de la disfonía en la calidad de vida. La aplicación de tales herramientas es parte de los elementos básicos para la valoración de las disfonías comunes ${ }^{3}$. Behlau ${ }^{4}$ sugiere que la aplicación de los protocolos de autoevaluación vocal debe ocurrir en tres momentos: en la anamnesis, en el transcurso de la terapia y al término de la rehabilitación vocal. Entre los protocolos disponibles, los más frecuentemente utilizados para evaluar el impacto de la disfonía en la calidad de vida de los pacientes con alteraciones vocales se muestran en la Tabla 1.

El voice handicap index (VHI 30) -y su versión abreviada (VHI 10)- es un instrumento utilizado para la valoración del menoscabo asociado a la disfonía que percibe el paciente, cuyas traducciones a la lengua española poseen validez y fiabilidad ${ }^{5,15}$. Su versión en español mantiene la sigla en inglés y consta de un protocolo de 30 ítems con 3 subescalas: dominio emocional, orgánico y funcional ${ }^{16}$.

El voice related quality of life, V-RQOL, es un protocolo de 10 ítems con dos dominios: socioemocional y físico. De aplicación rápida y práctica, presenta una relación del grado de disfonía con la calidad de vida del paciente con su queja vocal ${ }^{17}$. Fue elaborado por Hogikyan y Sethuraman ${ }^{7}$ en 1999. Los autores sugieren su aplicación antes de cualquier información u orientación vocal, incluso antes de la eva-

\begin{tabular}{|c|c|c|c|c|}
\hline Protocolo en inglés & Autor(es) & Inglés & $\begin{array}{c}\text { Traducción y } \\
\text { Adaptación en } \\
\text { Chile }\end{array}$ & $\begin{array}{l}\text { Validación } \\
\text { en Chile }\end{array}$ \\
\hline Voice handicap index $(\mathrm{VHI} 30)^{5}$ & Jacobson y cols. (1997) & & & \\
\hline Voice handicap index (VHI 10) 6 & Rosen y cols. (2004) & $\square$ & & \\
\hline Voice related quality of life (V-RQOL) ${ }^{7}$ & Hogikyan \& Sethuraman (1999) & & & \\
\hline Voice symptom scale - VoiSS ${ }^{8}$ & Deary y cols. (2003) & $\square$ & $\square$ & $\square$ \\
\hline Modern singer handicap index - MSHI & Fussi \& Fuschini T. (2008) & & & \\
\hline Singing voice handicap index - S-VHI ${ }^{10}$ & Cohen y cols. (2007) & $\square$ & & \\
\hline Pediatrics voice handicap index (P-VHI) ${ }^{11}$ & Zur y cols. (2007) & & & \\
\hline $\begin{array}{l}\text { Voice activity and participation profile } \\
\left(\text { VAPP) }{ }^{12}\right.\end{array}$ & Ma \& Yui (2001) & $\square$ & & \\
\hline $\begin{array}{l}\text { TVQMtF- Transsexual voice questionnaire } \\
\text { for male-to-female transsexuals. }{ }^{13}\end{array}$ & Dacakis y cols. (2013) & & & \\
\hline Reflux symptom index $(\mathrm{RSI})^{14}$ & Belafsky y cols. (2002) & $\square$ & & \\
\hline
\end{tabular}


luación. En el estudio publicado por Spina y cols. ${ }^{17}$ se constata que la calidad de vida del sujeto disfónico refleja las posibilidades físicas y emocionales en la vigencia de la disfonía.

El voice symptom scale-VoiSS, un instrumento que pretende ser un inventario de síntomas vocales y que funcionaría como línea de base, permitiendo observar los cambios de la disfonía del adulto en la clínica. Cuenta con una adaptación cultural y validación al español chileno publicado como ESV-CL ${ }^{18}$.

El voice activity and participation profile, $\mathrm{VAPP}^{12}$ es una herramienta de valoración de la calidad de vida relacionada con la salud que muestra propiedades psicométricas robustas ${ }^{19}$ y presenta cinco dimensiones: Autopercepción del problema de voz, emoción, comunicación diaria, comunicación social, Uso de la voz en el trabajo. De estas, las tres últimas no habían sido incorporadas en protocolos anteriores, agregando un foco diferente de evaluación en pacientes con disturbios vocales y profesionales de la voz. Además, la consignación de las preguntas difiere de otros protocolos, ya que es por medio de una escala analógica visual (EAV), una línea recta de $100 \mathrm{~mm}$ donde los extremos generalmente son acordados por ausencia o presencia del problema o alteración ${ }^{12}$. Este instrumento cuenta con versiones validadas en otros idiomas tales como portugués y español, conservando la cantidad de preguntas, ítems y forma de consignar las preguntas.

Con relación a grupos específicos, existen cuestionarios elaborados para población pediátrica, cantantes, transexuales y pacientes con reflujo laringofaríngeo. Para la población infantil, el pediatrics voice handicap index (P-VHI) es una herramienta confiable, váli$\mathrm{da}$, con buena consistencia interna y de fácil aplicación para pacientes pediátricos ${ }^{11}$. Fue traducida y validada en español de España, desarrollándose una versión exclusiva y acortada en este idioma de 10 ítems, conocida como P-VHI ${ }^{12,20}$.

Entender cómo los cantantes valoran la voz es un proceso fundamental en la evaluación vocal. El modern singer handicap index $(\mathrm{MSHI})^{9}$, creado en Italia, es un instrumento que entrega esta información y cuenta con adaptación al portugués y recientemente al español chileno ${ }^{21}$. El MSHI es un instrumento específico para cantantes populares que pre- senta 30 preguntas organizadas en tres ítems: discapacidad, deficiencia y minusvalía ${ }^{9}$. La versión original no cuenta con la validación, por lo que no presenta validaciones en otros idiomas. Por otro lado, el índice de incapacidad vocal para el canto (singing voice handicap index - S-VHI) está destinado a valorar el impacto en la salud producido por problemas de la voz en cantantes, y su traducción al español está validada, y presenta adecuada consistencia interna y fiabilidad ${ }^{22}$.

$\mathrm{El} \mathrm{TVQ} \mathrm{TH}^{\mathrm{MtF}}$ transsexual voice questionnaire for male-to-female transsexual - es un protocolo elaborado para individuos transexuales, traducido, con una adaptación transcultural y evaluación de las propiedades psicométricas del instrumento ${ }^{13}$. El TVQ ${ }^{\mathrm{MtF}}$ es un cuestionario indicado para profesionales que trabajan con feminización vocal, orientando y monitoreando la terapia vocal. Las validaciones culturales y del protocolo al idioma español facilitan la comprensión de la percepción vocal de transexuales (hombre a mujer) y el desarrollo de estrategias que mejoren la feminización vocal2 ${ }^{23}$.

Los pacientes con queja de reflujo laringofaríngeo también cuentan con un protocolo específico conocido por reflux symptom index (RSI) -en español, Sp-RSI, que es un protocolo de bajo costo de aplicación, confiable y válido, que puede ser empleado como un screening a estos pacientes ${ }^{24}$.

Los protocolos mencionados ofrecen información desde las percepciones del paciente disfónico, incluyendo sus observaciones y vivencias, rompiendo el binomio "presencia versus ausencia” de enfermedad. Además, ayudan a desarrollar la conciencia de los problemas vocales y verifican la evolución del tratamiento vocal realizado ${ }^{1}$. Por ejemplo, Smith y cols. ${ }^{25}$ mencionan en su estudio que pacientes disfónicos con menor puntaje en el VHI al inicio de la terapia presentan una tendencia a la adherencia a la terapia y éxito en su rehabilitación vocal.

En los últimos años se ha experimentado un aumento en el uso de protocolos de autoevaluación de calidad de vida, en su mayoría publicados en inglés. La necesidad de incorporar al proceso de evaluación vocal este tipo de instrumentos ha llevado a los profesionales a utilizar protocolos libremente traducidos al español, sin preocupación por la metodología. 
Tanto en el contexto clínico como de investigación, es relevante que los instrumentos empleados sean sometidos a un proceso de validación. Este consiste en adaptar culturalmente un instrumento al medio donde se quiere administrar y comprobar sus características psicométricas como: fiabilidad, validez, sensibilidad y factibilidad ${ }^{26}$. Ramada-Rodilla y cols. ${ }^{27}$ sugieren que el proceso de traducción, adaptación cultural y validación (TACV) sea exhaustivo, ya que la traducción simple puede conducir a una interpretación errónea debido a diferencias culturales y de lenguaje. En el caso de que la TACV no sea rigurosa, puede afectarse la precisión diagnóstica, el abordaje terapéutico, los registros epidemiológicos e, incluso, el diseño y puesta en marcha de políticas públicas. Cuando se señala que una herramienta ha sido sometida al proceso de validación quiere decir que el instrumento está adaptado culturalmente al medio en donde se quiere administrar y que sus cualidades psicométricas están comprobadas ${ }^{26}$.

Actualmente existe la tendencia de incorporar el punto de corte en estos instrumentos mediante curva ROC (receiver operating characteristics), pues el poder de análisis de esta medida permite identificar con mayor precisión la discriminación de los evaluadores en un sistema binario ${ }^{28}$. Además, el análisis de curva ROC visualiza la relación de los valores más altos entre la sensibilidad y especificidad, entendiéndose verdaderos positivos y falsos positivos respectivamente ${ }^{29}$. Combinado con los valores mayores de eficiencia y el producto, valor que comprueba la eficiencia de la prueba y precisión del resultado, se obtiene punto de corte $^{30}$. Este mismo estudio menciona que una de las características de mayor relevancia en la utilización de la curva ROC es la posibilidad de comparar los resultados con otras pruebas de diagnóstico. Sin embargo, aún hay pocos estudios de autoevaluación de la voz que han incorporado este tipo de análisis. Este artículo pretende ser una revisión narrativa de los instrumentos de autoevaluación empleados en la clínica vocal, que han sido adaptados culturalmente y validados al español en Chile.

Se definieron las palabras-clave del estudio, verificadas en los descriptores de salud DeCS y MeSH, a saber: voz; estudios de validación; calidad de vida; autoevaluación; Chile; com- paración transcultural. Se examinaron los sitios de búsqueda Pubmed, Scielo y Bireme usando las palabras clave en inglés. La revisión narrativa tuvo lugar el 17 de julio de 2020, usándose dos restricciones, relacionadas con el idioma (inglés y español) y considerando la fecha de publicación entre el 2008 y 2020 . El análisis de los resultados fue ejecutado por tres fonoaudiólogos expertos en voz.

Los artículos revisados fueron seleccionados según los criterios de inclusión al estudio. Se encontraron 41 artículos, eliminándose 24 artículos duplicados y 13 artículos de contenido no relacionado, resultando en 4 artículos analizados correspondientes a 3 instrumentos traducidos al español. Luego, se confeccionó la siguiente Tabla 2 referente a los protocolos de calidad de vida en voz traducidos al español, adaptados y/o validados en Chile.

\section{Discusión}

El uso de protocolos de autoevaluación que miden el impacto de la disfonía en la calidad de vida del individuo disfónico se ha masificado en los últimos años en la clínica vocal ${ }^{3}$. Se destaca que su uso ayuda a valorar las disfonías comunes. A su vez, se sugiere aplicarse en tres momentos: evaluación, transcurso de la terapia y alta, para mayor monitoreo de la consciencia vocal del paciente ${ }^{4}$. Smith y cols. ${ }^{25}$ refieren que la adherencia a la terapia y éxito en esta también se pueden verificar con el puntaje obtenido en estos protocolos.

Pero la mera aplicación del protocolo traducido y registro de resultados no es suficiente. Protocolos como el VHI y VHI-10 son ampliamente reconocidos y empleados en la clínica fonoaudiológica y otorrinolaringológica en Chile. Sin embargo, su aplicación puede conducir a error, una vez que este instrumento ha sido traducido, pero no ha completado la rigurosidad del proceso de $\mathrm{TACV}^{27}$. Sus propiedades psicométricas no se encuentran verificadas haciendo cuestionable la interpretación de puntajes obtenidos en población chilena.

Propuestas tales como "consensus based standards for the selection of health measurement instruments, COSMIN", sugieren recomendaciones para el proceso de traducción, adaptación cultural y validación de protocolos ${ }^{33}$. Lo 
Tabla 2. Protocolos de calidad de vida en voz traducidos, adaptados y validados en Chile

\begin{tabular}{|c|c|c|}
\hline Autores & Artículo & Investigación desarrollada \\
\hline $\begin{array}{l}\text { Ruston FC, } \\
\text { Moreti F, } \\
\text { Vivero M, } \\
\text { Malebran C, } \\
\text { Behlau } \mathrm{M}^{18}\end{array}$ & $\begin{array}{l}\text { Cross-cultural } \\
\text { adaptation of the } \\
\text { Chilean version } \\
\text { of the Voice } \\
\text { Symptom Scale- } \\
\text { Voiss }\end{array}$ & $\begin{array}{l}\text { La traducción del voice symptom scale-VoiSS para el } \\
\text { español chileno, siguió la recomendación del SAC } \\
\text { (Scientific Auditory Commitee Outcome Trust). El } \\
\text { instrumento fue denominado escala de síntomas } \\
\text { vocales-ESV, compuesta de } 30 \text { preguntas y } 5 \text { respues- } \\
\text { tas. Con el objetivo de lograr la equivalencia cultural, } \\
\text { la ESV fue aplicada a } 15 \text { individuos con problemas } \\
\text { vocales, como grupo control. Las discrepancias fueron } \\
\text { solucionadas y la ESV modificada fue aplicada a tres } \\
\text { personas más con problemas vocales, no encontrán- } \\
\text { dose preguntas incomprendidas o inapropiadas para } \\
\text { la cultura chilena. La ESV refleja la versión original de } \\
\text { inglés, tanto en la cantidad de preguntas como en la } \\
\text { limitación de los dominios emocional y físico }\end{array}$ \\
\hline
\end{tabular}

Contreras F,

Moreti F,

Malebran C,

Viveros $\mathrm{M}$,

Behlau $\mathrm{M}^{31}$

\section{(2018)}

Cross-cultural adaptation, validation, and cutoff values of the Chilean version of the voice symptom scale: Voiss
Se evaluaron las propiedades de la escala de síntomas de la voz (VoiSS)-chileno versión en español, cumpliendo todos los criterios del comité científico asesor del medical outcomes trust (SAC), evaluando la validez, empleando criterios externos (excelente, muy bueno, regular y pobre). La fiabilidad fue realizada por test-retest. Además, aplico una evaluación perceptual pre y postratamiento de 12 pacientes con escala GRBAS. El protocolo chileno español adaptado de VoiSS, Escala de síntomas vocales (ESV-CL), se aplicó a 205 sujetos, 89 con disfonía y 116 de ellos vocalmente sanos. La ESV-CL demuestra una alta validez, fiabilidad y capacidad de respuesta. Un puntaje de corte de 35,5 fue determinado utilizando la alta sensibilidad (90\%) y la especificidad (75\%) encontradas en sujetos con disfonía en comparación con a sujetos vocalmente sanos, mediante curva ROC. La versión chilena revela que es culturalmente equivalente a la versión original, valido para su utilización en la población chilena

MSHI fue traducido como índice de desventaja vocal para canto popular-IDVCP-Ch, compuesto de 30 ítems y cuatro tipos de respuestas. Fue aplicado a 25 cantantes populares, amateurs y profesionales. Considerándose las adaptaciones necesarias al idioma y cultura, se logró la traducción y la equivalencia cultural, reflejando la versión original de italiano, tanto en la cantidad de ítems como en las limitaciones de los dominios discapacidad, desventaja e impedimentos

Contreras $\mathrm{F}$, González N, Vivero M, Guzmán $\mathrm{M}^{32}$

(2018)

Cross-cultural

adaptation of the

Chilean version

of the modern

singing handicap index: MSHI
(2019)

Equivalencia cultural de la versión chilena del voice-related quality of life

( $\mathrm{V}$-RQOL)
La traducción del instrumento $\mathrm{V}$-RQOL, siguió las recomendaciones del SAC. El instrumento fue renombrado como "Medición de la Calidad de Vida en Relación a la Voz" (MCV-RV)", con 10 preguntas y 5 opciones de respuesta. Para la equivalencia cultural, se aplicó el protocolo a 15 personas con trastornos vocales, evaluándose sus respuestas. La versión final del protocolo MCV-RV refleja la versión original en inglés, tanto en número como en tipos de preguntas, incluido el funcionamiento físico y aspectos socioemocionales

\section{Conclusión}

Existe una equivalencia cultural del Voiss para el español chileno, que recibe el nombre de ESV. La validación de la ESV para el español chileno está en proceso de conclusión

Validación del ESV fue lograda para el español chileno

Fue demostrada la equivalencia intercultural y lingüística al español chileno del IDVCP-Ch

Fue lograda la adaptación cultural de V-RQOL para el español chileno (MCV-RV). La validación del instrumento se encuentra en curso para el español de Chile 
que concuerda con autores que mencionan que el proceso de TACV de protocolos a cada país, mantenga la fiabilidad de resultados arrojados, reflejando su cultura ${ }^{27}$. Ejemplo de lo anterior, es el caso del VoiSS y sus versiones en español, cuya adaptación y validación chilena desde un punto de vista cultural, no es adecuada para aplicación a la población española ${ }^{34}$.

En esta investigación, la búsqueda arrojó cuatro artículos, relacionados a tres protocolos, los cuales están adaptados culturalmente al español de Chile (voice symptom scale-VoiSS o escala de síntomas vocales, ESV-CL; modern singer handicap index-MSHI y el V-RQOL, con el nombre medición de la calidad de vida en relación a la voz, o MCV-RV ${ }^{18,21,32}$.

\section{Conclusión}

El objetivo de esta revisión narrativa fue identificar los instrumentos de autoevaluación empleados en la clínica vocal, que han sido adaptados culturalmente y validados al español en Chile. El uso de protocolos en la clínica son parte fundamental para la valoración vocal, aportando al especialista el nivel de consciencia que el sujeto con alteraciones vocales presenta en relación con su voz.

Apelando al rigor en la clínica vocal, se debiera emplear el único protocolo que cumple con las recomendaciones de COSMIN y SAC, y que corresponde a la versión chilena del VoiSS, la escala de síntomas vocales - ESV. En su proceso de TACV cumple con todos los requisitos y criterios internacionales establecidos, contribuyendo así para la clínica vocal ${ }^{31}$. Como se constata con esta investigación, la cantidad de protocolos de autoevaluación vocal en Chile con traducción, adaptación cultural y validación estandarizada continúa siendo escasa. Existe una tarea pendiente en la fonoaudiología chilena en relación a los demás protocolos.

\section{Bibliografía}

1. Roy N, Barkmeier-Kraemer J, Eadie T, et al. Evidence-based clinical voice assessment: a systematic review. Am J Speech Lang Pathol. 2013;22(2):212-226. doi: 10.1044/1058-0360(2012/12-0014).
2. American Speech Hearing Association. Voice Disorders. S.f. https:// www.asha.org/PRPSpecificTopic. aspx? folderid $=8589942600 \&$ section $=$ Assessment

3. Dejonckere PH, Bradley P, Clemente P, et al. A basic protocol for functional assessment of voice pathology, especially for investigating the efficacy of (phonosurgical) treatments and evaluating new assessment techniques. Guideline elaborated by the Committee on Phoniatrics of the European Laryngological Society (ELS). Eur Arch Otorhinolaryngol. 2001;258(2):77-82. doi: 10.1007/ s004050000299.

4. Behlau M. ed. Voz: o livro do especialista. Rio de Janeiro: Thieme Revinter, 2001.

5. Jacobson BH, Johnson A, Grywalski C, et al. The Voice Handicap Index (VHI): Development and Validation. Am J Speech Lang Pathol. 1997;6(3):66-70. doi: 10.1044/1058-0360.0603.66.

6. Rosen CA, Lee AS, Osborne J, Zullo T, Murry T. Development and Validation of the Voice Handicap Index-10: The Laryngoscope. 2004;114(9):1549-1556. doi: 10.1097/00005537-200409000-00009.

7. Hogikyan ND, Sethuraman G. Validation of an instrument to measure voice-related quality of life (V-RQOL). J Voice. 1999;13(4):557-569. doi: 10.1016/ s0892-1997(99)80010-1.

8. Deary IJ, Wilson JA, Carding PN, MacKenzie K. VoiSS. Journal of Psychosomatic Research. 2003;54(5):483-489. doi: 10.1016/S00223999(02)00469-5

9. Fussi F, Fuschini T. Foniatria artistica: la presa in carico foniatrico-logopedica del cantante classico e moderno. Audiol Foniatr ;13(1-2):4-28. 2008;13(12):4-28.

10. Cohen SM, Jacobson BH, Garrett CG, et al. Creation and Validation of the Singing Voice Handicap Index. Ann Otol Rhinol Laryngol. 2007;116(6):402-406. doi: 10.1177/000348940711600602

11. Zur KB, Cotton S, Kelchner L, Baker S, Weinrich B, Lee L. Pediatric Voice Handicap Index (pVHI): a new tool for evaluating pediatric dysphonia. Int J Pediatr Otorhinolaryngol. 2007;71(1):77-82. doi: 10.1016/j. ijporl.2006.09.004.

12. Ma EP, Yiu EM. Voice activity and participation profile: assessing the impact of voice disorders on daily activities. J Speech Lang Hear Res. 2001;44(3):511-524. doi: 10.1044/10924388(2001/040).

13. Dacakis G, Davies S, Oates JM, Douglas JM, Johnston JR. Development and Preliminary Evaluation of the Transsexual Voice Questionnaire for Male-to-Female Transsexuals. Journal of Voice. 2013;27(3):312-320. doi: 10.1016/j.jvoice.2012.11.005.

14. Belafsky PC, Postma GN, Koufman JA. Validity and Reliability of the Reflux Symptom Index (RSI). Journal of Voice. 2002;16(2):274-277. doi: 10.1016/ 
S0892-1997(02)00097-8.

15. Núñez-Batalla F, Corte-Santos P, Señaris-González B, Llorente-Pendás JL, Górriz-Gil C, Suárez-Nieto C. Adaptación y validación del índice de incapacidad vocal (VHI-30) y su versión abreviada (VHI-10) al español [Adaptation and validation to the Spanish of the Voice Handicap Index (VHI-30) and its shortened version (VHI-10)]. Acta Otorrinolaringol Esp. 2007; 58(9):386-392.

16. Costa T, Oliveira G, Behlau M. Validation of the Voice Handicap Index: 10 (VHI-10) to the Brazilian Portuguese. Codas. 2013;25(5):482-485. doi: 10.1590/ S2317-17822013000500013.

17. Spina AL, Maunsell R, Sandalo K, Gusmão R, Crespo A. Correlação da qualidade de vida e voz com atividade profissional. Revista Brasileira de Otorrinolaringologia. 2009; 75(2):275-279. doi: 10.1590/S0034-72992009000200019.

18. Ruston FC, Moreti F, Vivero M, Malebran C, Behlau M. Cross-cultural adaptation of the Chilean version of the Voice Symptom Scale VoiSS. Codas. 2016;28(5):625-633. doi: 10.1590/23171782/20162015249.

19. Fava G, Paolillo NP, Oliveira G, Behlau M. Crosscultural adaptation, validation, and cutoff point of the Italian version of the voice activity and participation profile: Profilo di Attività e Partecipazione Vocale. J Voice. 2015;29(1):130.e11-130.e1.3E19. doi: 10.1016/j. jvoice.2014.04.008.

20. Sanz L, Bau P, Arribas I, Rivera T. Development and validation of a short version of the Spanish pediatric voice handicap index (P-VHI-10). Int J Pediatr Otorhinolaryngol. 2016;88:113-116. doi: 10.1016/j. ijporl.2016.06.053.

21. Correa S, Contreras F, Castillo A, Moreti F, Behlau M. Cross-cultural adaptation of the Chilean version of the Modern Singing Handicap Index: MSHI. Equivalencia cultural de la versión Chilena del Modern Singing Handicap Index: MSHI. Codas. 2018;30(3):e20170156. doi: 10.1590/2317$1782 / 20182017065$.

22. García-López I, Núñez-Batalla F, Gavilán Bouzas J, Górriz-Gil C. Validación de la versión en español del índice de incapacidad vocal (S-VHI) para el canto [Validation of the Spanish version of the voice handicap index for vocal singing (SVHI)] [published correction appears in Acta Otorrinolaringol Esp. 2010 Nov-Dec;61(6):469]. Acta Otorrinolaringol Esp. 2010;61(4):247-254. doi: 10.1016/j.otorri.2010.01.012.

23. Mora E, Carrillo A, Giribet A, Becerra A, Lucio MJ, Cobeta I. Translation, Cultural Adaptation, and Preliminary Evaluation of the Spanish Version of the
Transgender Voice Questionnaire for Male-to-Female Transsexuals (TVQ ${ }^{\mathrm{MtF}}$ ). J Voice. 2018;32(4):514.e1514.e6. doi: 10.1016/j.jvoice.2017.05.012.

24. Calvo-Henríquez C, Ruano-Ravina A, Vaamonde $\mathrm{P}$, et al. Translation and Validation of the Reflux Symptom Index to Spanish. J Voice. 2019;33(5):807. e1-807.e5. doi: 10.1016/j.jvoice.2018.04.019.

25. Smith BE, Kempster GB, Sims HS. Patient factors related to voice therapy attendance and outcomes. $J$ Voice. 2010;24(6):694-701. doi: 10.1016/j. jvoice.2009.03.004.

26. Carvajal A, Centeno C, Watson R, Martínez M, Sanz Rubiales Á. ¿Cómo validar un instrumento de medida de la salud? Anales del Sistema Sanitario de Navarra. 2011; 34(1):63-72.

27. Ramada-Rodilla JM, Serra-Pujadas C, \& DelclósClanchet GL. Adaptación cultural y validación de cuestionarios de salud: Revisión y recomendaciones metodológicas. Salud Pública de México. 2013; 55(1):57-66.

28. Fawcett T. An introduction to ROC analysis. Pattern Recognition Letters. 2006; 27(8):861-874. doi: 10.1016/j.patrec.2005.10.010.

29. Burgueño M, García-Bastos J, González-Buitrago JM. Las curvas ROC en la evaluación de las pruebas diagnósticas. Medicina Clínica. 1995; 104(17):661670 .

30. Behlau M, Madazio G, Moreti F, et al. Efficiency and Cutoff Values of Self-Assessment Instruments on the Impact of a Voice Problem. J Voice. 2016;30(4):506. e9-506.e18. doi: 10.1016/j.jvoice.2015.05.022.

31. Contreras F, Moreti F, Malebran C, Viveros M, Behlau M. Cross-Cultural Adaptation, Validation, and Cutoff Values of the Chilean Version of the Voice Symptom Scale: VoiSS 2018; 47th Annual Symposium Care of the Professional Voice, Philadelphia.

32. Contreras F, Gonzalez N, Vivero M, \& Guzman M. Equivalencia cultural de la Versión Chilena del Voice-Related Quality of Life (V-RQOL). CoDAS. 2019; 31(3):e20180213. doi: 10.1590/23171782/20192018213.

33. Mokkink LB, Terwee CB, Patrick DL, et al. The COSMIN study reached international consensus on taxonomy, terminology, and definitions of measurement properties for health-related patientreported outcomes. J Clin Epidemiol. 2010;63(7):737745. doi: 10.1016/j.jclinepi.2010.02.006.

34. Velandrino A, Cabello F, Parra M, Nicolás MJ, Losana E. Adaptación cultural y estudio piloto de la versión española de la Voice Symptom Scale (VoiSS). Revista de Logopedia, Foniatría y Audiología. 2018;38(4):143147. doi: 10.1016/j.rlfa.2018.07.003. 